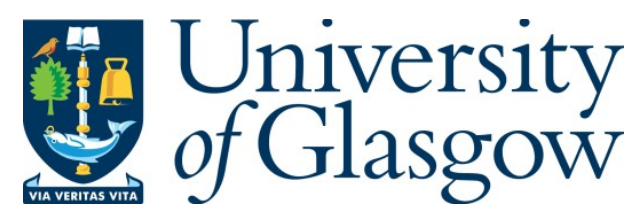

Fioranelli, F., Griffiths, H., and Ritchie, M. (2015) Personnel recognition based on multistatic micro-Doppler and singular value decomposition features. Electronics Letters, 51(25), pp. 2143-2145.

There may be differences between this version and the published version. You are advised to consult the publisher's version if you wish to cite from it.

This paper is a postprint of a paper submitted to and accepted for publication in Electronics Letters and is subject to Institution of Engineering and Technology Copyright. The copy of record is available at IET Digital Library.

http://eprints.gla.ac.uk/118190/

Deposited on: 29 April 2016

Enlighten - Research publications by members of the University of Glasgow http://eprints.gla.ac.uk 


\section{Personnel Recognition Based on Multistatic Micro-Doppler and Singular Value Decomposition Features}

\author{
F. Fioranelli, M. Ritchie, and H. Griffiths
}

This letter discusses the use of micro-Doppler signatures experimentally collected by a multistatic radar system to recognize and classify different people walking. A suitable feature based on Singular Value Decomposition of the spectrograms is proposed and tested with different types of classifiers. It is shown that high accuracy between $97-99 \%$ can be achieved when multistatic data are used to perform the classification.

Introduction: It is well known that moving humans present additional modulations on top of their main Doppler shift because of the motion of limbs and body known as micro-Doppler [1]. These microDoppler signatures have been used to discriminate between human and non-human targets such as animals (dogs, horses) and vehicles [2], to distinguish between different activities such as running, crawling, or walking [3], and to characterize free or confined movement of arms related to carrying objects, potentially weapons [4-5]. It has been shown that the classification accuracy can be degraded when the aspect angle between the target velocity vector and the radar line-of-sight is higher than $30^{\circ}$ and even more if close to $90^{\circ}$, and bistatic or multistatic systems have been proposed to mitigate this problem. [2,6]. There is however little work in the literature to investigate the use of human micro-Doppler signatures for identification and recognition of different people performing the same activity. This is expected to be a challenging task as targets of the same type are not too dissimilar from one another, hence very robust features are required for successful classification. Features based on the Cadence Velocity Diagram of human micro-Doppler signatures have been proposed in [7] to discriminate between four different subjects walking and running using data recorded with a $\mathrm{CW} \mathrm{X}$-band monostatic radar. Classification accuracy above $90 \%$ were reported in this indoor controlled test, where the subjects were moving on a treadmill.

In this letter we investigate the use of Singular Value Decomposition (SVD) to extract suitable features for recognition and classification of different subjects walking. The experimental data were recorded in an open field using a multistatic radar and the subjects were realistically walking forward towards the radar. The effect on recognition performance of different approaches in combining multistatic information is discussed, and different types of classifiers are tested. SVD-based features were already used in our previous work [8] to discriminate between armed and unarmed personnel, and they have been reported in the classification of different types of micro-drones [9]. The feature extraction approach proposed in this letter is different from the aforementioned references, as it assumes that the relevant information is not concentrated in only a few singular vectors, but in the whole matrices $\mathrm{U}$ and $\mathrm{V}$ derived from the SVD. High classification accuracy above $98 \%$ is achieved with the single SVD-based feature proposed in this letter.

Experimental setup and radar system: The data presented in this paper were collected using the University College London multistatic radar system NetRAD [5]. NetRAD is a coherent pulsed radar consisting of three separate but identical nodes that operate at $2.4 \mathrm{GHz}$, $\mathrm{S}$-band. The transmitted power was approximately $+23 \mathrm{dBm}$, with vertically polarized antennas with $24 \mathrm{dBi}$ gain and approximately $10^{\circ} \times 10^{\circ}$ beam-width. The RF parameters chosen for the experiment described in this paper were linear up-chirp modulation with $45 \mathrm{MHz}$ bandwidth and $0.6 \mu$ s duration, $5 \mathrm{kHz}$ pulse repetition frequency (PRF) which allows the whole human micro-Doppler signature to be contained in the unambiguous Doppler region, and $5 \mathrm{~s}$ duration of each recording to collect multiple periods of the average human walking gate. The experiment took place in December 2014 in an open field at the UCL Sports Ground. Fig. 1 shows the geometry of the experiment with the three NetRAD nodes deployed along a linear baseline with approximately $40 \mathrm{~m}$ inter-node separation and the person at approximately $70 \mathrm{~m}$ from the linear baseline. Node 3 was used as monostatic transceiver, with Node 2 and Node 1 as bistatic receivers, with resulting bistatic angles equal to approximately $30^{\circ}$ and $60^{\circ}$. Three different subjects took part in the experiment and walked towards the middle node, as shown in Fig. 1. The key body parameters of the three subjects were $1.87 \mathrm{~m}, 90 \mathrm{~kg}$, average body type for person $1,1.70 \mathrm{~m}, 69$ $\mathrm{kg}$, average body type for person 2 , and $1.77 \mathrm{~m}, 65 \mathrm{~kg}$, slim body type for person 3. The total number of recorded datasets was therefore 45 , assuming 3 subjects, 5 repetitions of the movement, and 3 nodes.

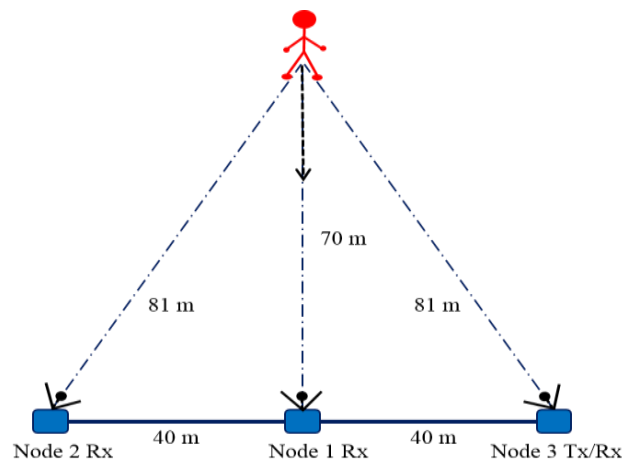

Fig. 1 Geometry of the experimental setup

Data analysis and classification: The recorded data were processed using Short Time Fourier Transform (STFT) to characterize the human micro-Doppler signatures. The STFTs were calculated using $0.3 \mathrm{~s}$ Hamming windows with $95 \%$ overlap. Fig. 2 shows examples of microDoppler signatures, one for each of the three subjects, using data from the monostatic node. It can be seen that there are visible differences in the signatures from different subjects, in particular in the periodic peaks at higher and lower Doppler due to the movement of the limbs. These differences can be quantified in numerical features for automatic classification. The spectrograms were then divided in $1 \mathrm{~s}$ long blocks generating 225 blocks, i.e. five times the total number of recorded datasets. SVD decomposition was applied on each block to extract suitable features. As mentioned in the introduction, the whole matrices derived from SVD are considered for feature extraction rather than individual singular vectors. The sum of the intensity of the elements of the matrix $U$ is found to be a particular effective feature, hence there are 225 feature samples to be used as inputs to the classifiers. Fig. 3 shows feature samples for the three different subjects as extracted from data collected at the three radar nodes. A good inter-class separation can be seen, hence good classification performance is expected using this feature.

Different types of classifiers have been tested with these data, namely linear discriminant analysis (LDA), quadratic discriminant analysis (QDA), diagonal-linear discriminant analysis (DLDA), diagonal-quadratic discriminant analysis (DQDA), naïve Bayes with kernel functions estimators (NB), nearest neighbours with 3 samples (NN3) and 5 samples (NN5), and a classification tree (CT). The aim is to investigate any difference in the classification performance with different classifiers, and their efficiency in terms of processing time when implemented in MATLAB. A more detailed description of the classifiers can be found in [10]. Each classifier was trained with $20 \%$ of the available feature samples, and the remaining samples were used to evaluate the performance and calculate the error as the total number of misclassification events over the total number of samples. Each classifier has been tested 100 times with random changes in the set of samples used for training to test the consistency of the classifiers' behaviours, and the average error over these repetitions was calculated. The results reported here are expressed in terms of percentage accuracy calculated as $100 \%$ minus this error.

Three different ways of combining multistatic data were tested and compared with the use of monostatic data only, as for a conventional radar. In the first approach feature samples from all radar nodes are processed by a centralized single classifier providing the final decision. In the second approach separate classifiers process the features samples at each radar node providing partial decisions, which are then combined in a final decision through a voting procedure, i.e. the final decision has to get a majority of two out of three nodes. The third approach takes into account the level of confidence of each partial decision with a threshold. If two nodes agree on a partial decision and both have higher 
confidence than the threshold, then they will provide the final decision. However if one of these two nodes has lower confidence than the threshold, and if at the same time the third node has a higher level of confidence than the other two nodes, then the final decision will be provided by the third node. This approach aims at preventing that two nodes with low level of confidence may lead to a misclassification event. The threshold has been set at $65 \%$ as the value providing the best classification results after testing values in the interval $55-75 \%$ with the available data. In both the second and third approach, if there is no partial decision reached by at least two radar nodes, the final decision is simply taken by the node with the highest level of confidence.

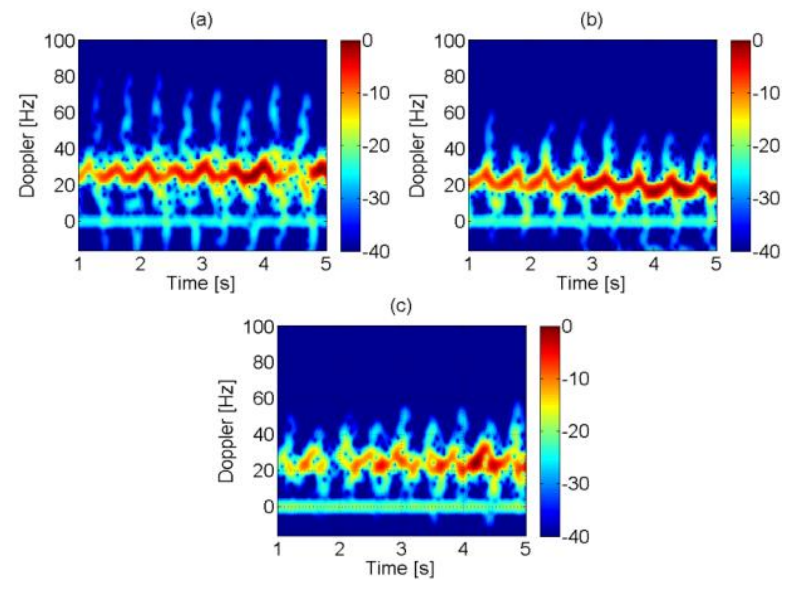

Fig. 2 Micro-Doppler signatures extracted from monostatic data for subject $1(a)$, subject $2(b)$, and subject $3(c)$
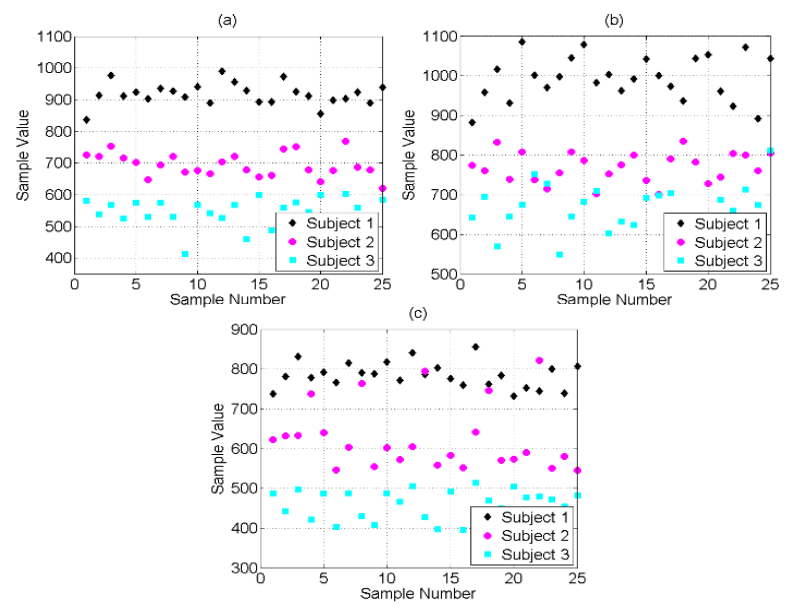

Fig. 3 SVD-based feature samples for three subjects extracted from node 1 data (a), node 2 data (b), and node 3 data (c)

Table 1 shows the classification accuracy for different classifiers and approaches in using multistatic data, as well as the required processing time for each classifier. The accuracy is consistently above 95\% using only monostatic data, and increases up to an average of $99 \%$ when multistatic data are used with the binary voting or threshold voting approach at separate classifiers. It is interesting to notice that the accuracy decreases when the multistatic data are used at a centralized classifier. This was already observed in our previous work in [5, 8]. The fastest classifiers appear to be the classification tree, the nearestneighbours, and the Naîve Bayes, whereas the discriminant analysis appears to be slower, even requiring twice the time in its quadratic variants. The memory usage for different classifiers was also investigated, but did not exhibit significant variations with different classifiers, as the values varied only between 1840-1860 MB. It should be noted that processing time and memory usage were tested on a desktop Windows workstation in the same conditions for each classifier, and the code had a basic implementation, not aiming at optimization.

Conclusion: This letter has presented the use of micro-Doppler signatures recorded by a multistatic radar system to recognize and identify different people walking. A feature based on the whole matrix $\mathrm{U}$ derived from the SVD decomposition of the spectrograms has been proposed. It has been shown that high classification accuracy above 98\% can be achieved when multistatic data are combined using separated classifiers at each radar node. The proposed approach has been shown to be robust with different types of classifiers, hence the most computationally efficient ones can be used. Future work will aim at testing this feature for a wider number of subjects, activities, and deployment geometries of the multistatic radar.

TABle 1: Percentage classification accuracy for different types of classifiers and approaches in using multistatic data, and processing time in seconds

\begin{tabular}{|c|c|c|c|c|c|}
\hline $\begin{array}{c}\text { Classifier } \\
\text { Types }\end{array}$ & $\begin{array}{c}\text { Mono } \\
\text { data } \\
\text { only }\end{array}$ & $\begin{array}{c}\text { All } \\
\text { multi } \\
\text { data }\end{array}$ & $\begin{array}{c}\text { Binary } \\
\text { voting }\end{array}$ & $\begin{array}{c}\text { Threshold } \\
\text { voting }\end{array}$ & $\begin{array}{c}\text { Processing } \\
\text { time }[\mathbf{s}]\end{array}$ \\
\hline LDA & 98.9 & 72.2 & 98.8 & 99.4 & 6.651 \\
\hline QDA & 97.6 & 72.2 & 98.9 & 98.9 & 8.630 \\
\hline DLDA & 98.9 & 72.2 & 98.8 & 99.4 & 6.665 \\
\hline DQDA & 97.6 & 72.2 & 98.9 & 98.9 & 8.648 \\
\hline NB & 95.4 & 72.1 & 97.9 & 97.5 & 4.308 \\
\hline NN3 & 98.7 & 71.1 & 99.4 & 99.4 & 4.223 \\
\hline NN5 & 98.7 & 70.9 & 99.1 & 99.4 & 4.254 \\
\hline CT & 98.8 & 71.3 & 99.6 & 99.6 & 4.137 \\
\hline
\end{tabular}

Acknowledgments: This work was funded by the IET A F Harvey Prize awarded to Prof Hugh Griffiths (2013). The authors are grateful to Frack Wei and Saad Alhuwaimel for their help in the experimental trial.

F. Fioranelli, M. Ritchie, and H. Griffiths (Department of Electronic and Electrical Engineering, UCL, London, UK)

E-mail: f.fioranelli@ucl.ac.uk

\section{References}

1. Chen, V.C., Tahmoush, D., Miceli, W.L., 'Radar Micro-Doppler Signatures: Processing and Applications', Institution of Engineering and Technology, 2014.

2. Youngwook, K., Sungjae, H., Jihoon, K., 'Human Detection Using Doppler Radar Based on Physical Characteristics of Targets', IEEE Geoscience and Remote Sensing Letters, 2015, 12, (2), pp. 289-293.

3. Youngwook, K., Hao, L., 'Human Activity Classification Based on Micro-Doppler Signatures Using a Support Vector Machine', IEEE Trans. Geoscience and Remote Sensing, 47, (5), pp. 1328-1337, 2009.

4. Orović, I., Stanković, S., Amin, M., 'A New Approach for Classification of Human Gait Based on Time-Frequency Feature Representations', Signal Processing, 91, (6), pp. 1448-1456, 2011.

5. Fioranelli, F., Ritchie, M., Griffiths, H., 'Multistatic Human MicroDoppler Classification of Armed/Unarmed Personnel', IET Radar, Sonar \& Navigation, vol. 9, pp. 857-865, 2015.

6. Fairchild, D.P., Narayanan, R.M., 'Determining Human Target Facing Orientation Using Bistatic Radar Micro-Doppler Signals', Proc. SPIE 9082, Active and Passive Signatures V, 908203 (June 4, 2014).

7. Ricci, R., Balleri, A., 'Recognition of humans based on radar microDoppler shape spectrum features', IET Radar, Sonar \& Navigation, accepted for publication and available online.

8. Fioranelli, F., Ritchie, M., Griffiths, H., 'Classification of Unarmed/Armed Personnel Using the NetRAD Multistatic Radar for Micro-Doppler and Singular Value Decomposition Features', IEEE Geoscience and Remote Sensing Letters, vol.12, no.9, pp.1933-1937, Sept. 2015.

9. De Wit, J.J.M., Harmanny, R., Molchanov, P., 'Radar MicroDoppler Feature Extraction Using the Singular Value Decomposition', 2014 International Radar Conference, October 2014, Lille, France.

10. Hastie, T., Tibshirani, R., Friedman, J., 'The Elements of Statistical Learning: Data Mining, Inference, and Prediction', Springer Series in Statistics, 2009. 